\title{
NUMERICAL STUDY ON CAPABILITIES CONSTRUCTIONS OF ELECTROMAGNETIC ACOUSTIC TRANSDUCERS (EMAT) WITH PERMANENT MAGNETS FOR INLINE INSPECTION OF PIPELINE 6"
}

\author{
Lazarev Igor \\ Moscow Power Engineering Institute (national reseach university) ,Krasnokazarmennaya st.14, \\ Moscow 111250, Russian Federation
}

\begin{abstract}
Pipeline operators are paying particular attention to the integrity of pipes. Currently the most common methods used for in-line pipeline inspection is Magnetic Flux Leakage (MFL) and ultrasound techniques. EMAT method is a new method, based on a generation of ultrasonic waves. Traditional ultrasonic technologies (UT) based on piezoelectric transducers are only applicable with liquid couplant between the transducer and the pipe wall. EMAT design typically consists of a system of permanent magnets and the conductor. Induced currents interact with the magnetic field of the permanent magnets create a Lorentz force in the testing object, causing ultrasonic waves. EMA method does not require the presence of fluid between the transducer and the object under test. Also, only EMAT system allows detecting stress corrosion cracking (SCC), to predict the future cracks.
\end{abstract}

Keywords: EMAT, in-line inspection, pipeline inspection, non-destructive testing
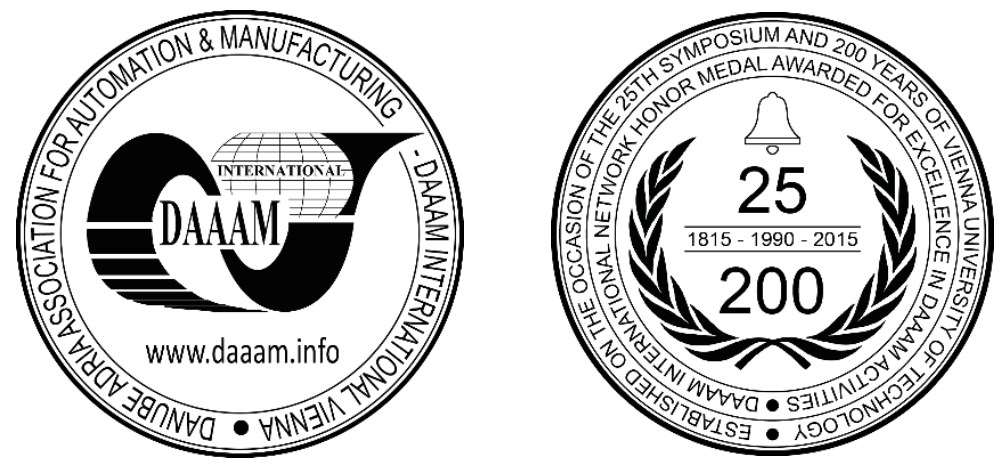

This Publication has to be referred as: Lazarev, I[gor] (2016). Numerical study on capabilities constructions of electromagnetic acoustic transducers (EMAT) with permanent magnets for inline inspection of pipeline 6", Proceedings of the 26th DAAAM International Symposium, pp.0936-0939, B. Katalinic (Ed.), Published by DAAAM International, ISBN 978-3-902734-07-5, ISSN 1726-9679, Vienna, Austria

DOI: 10.2507/26th.daaam.proceedings.131 


\section{Introduction}

EMAT technology relates to the field of ultrasonic non-destructive testing. As compared to traditional piezoelectric transducers, EMAT systems have a number of important advantages: does not require fluid contact between the transducers and the object of control (suitable for diagnostic of heated bodies), does not require surface preparation of the object under control, generation of ultrasonic waves occurs in control sample. EMAT method can only be implemented in the conductive media, but such diagnostic is suitable for gas and oil pipelines inspection since they are made of steel alloys [1].

The operating principle of such converters is the occurrence of the Lorentz force in the testing object fig.1. The construction of transducer consists of a permanent magnets and a coil arranged from the surface of the inspection object at a distance. High frequency current pulse was used to drive the meander coil, inducing currents in the sample. Induced currents interact with the static magnetic field, and as a result, there is the Lorentz force (1)

$$
\overrightarrow{F_{l}}=\overrightarrow{F_{l}} \times \vec{B}
$$

Generally, the force occurring in the control sample is dependent on the Lorentz force and the magnetostriction. In early studies noted that the effect of magnetostriction effect is negligible [2].

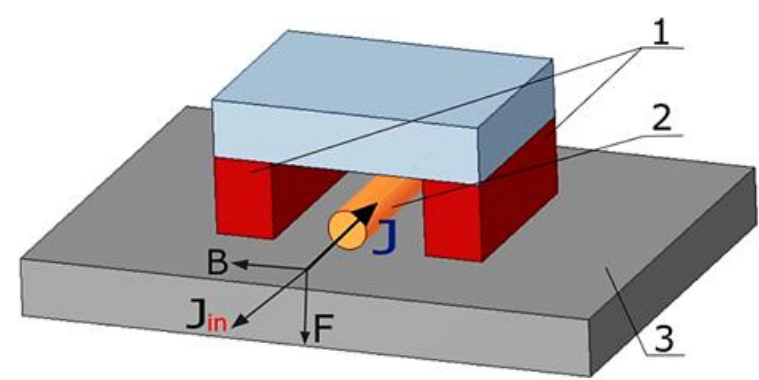

Fig.1. Construction of electromagnetic acoustic transducer: 1- permanent magnetic system, 2- coil, 3 - test object.

Depending on the mutual position of the inductor and the constant filed, different constructions of EMA transducer can be design and various modes of ultrasonic waves can be generated.

One of the common problems of EMAT systems is to ensure a sufficient level of static magnetic field in the object of control. From sources we know, that the necessary results can be considered as the level of magnetic flux density of more than $1 \mathrm{Tl}$ [2]. The researchers also point out the following problems appearing in such transducers: skin effect, acoustic manifestation of Barkhausen effect in ferromagnetic metals, appearance of ponderomotive forces [3].

\section{Magnetic field}

Providing a sufficient level of static magnetic field is an important issue in the development of EMA transducers. Some authors propose to use coils only EMAT concept [4], without magnets. A pipeline transportation service is of great length, in this regard, in-line inspection is a great solution for early monitoring the integrity of pipes; the sensor is moved inside the pipe in the flow of the pumped product, allowing you to control the long-distance pipes. Apply permanent magnets to provide static magnetic field is a best choice for in-line inspection. The sensor must ensure the unimpeded passage of all sections of the pipeline; in this case, in-line inspection has a significant dimensional limit for scan tools. Saturation of the magnetic core is a special problem of this diagnostic equipment for the inspection of pipes 6".

COMSOL Multiphysics software to calculate model EMAT sensor was used. The first model is a simple configuration of EMAT sensor fig.2. Magnetic core is steel $175.4 \mathrm{~mm}$ in length and $40.5 \mathrm{~mm}$ in width with high saturation magnetic flux density (over $2.2 \mathrm{Tl}$ ), NdFeB permanent magnets with remanent flux density $1.32 \mathrm{Tl}$, tube is steel 1020 wall thickness $9 \mathrm{~mm}$, cooper coil with $5 \mathrm{~mm}$ in length and $2 \mathrm{~mm}$ in width with a lift-off distance $3 \mathrm{~mm}$ to the tube.

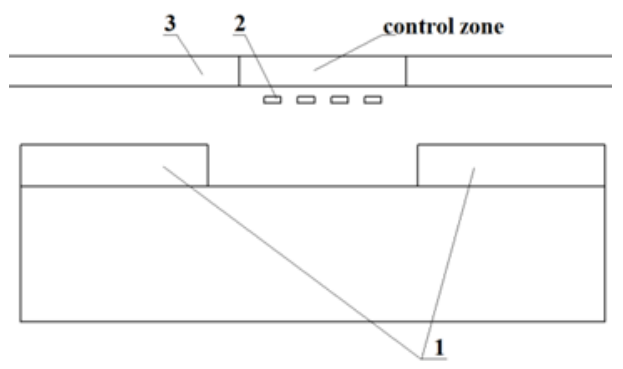

Fig.2. 2D COMSOL simple model EMAT sensor: 1 - permanent magnets, 2-meander coil, 3- steel pipe. 
At magnetic field application a static magnetic field a two 2D axisymmetric models was calculated. At this paper a two configurations of magnetic system of EMAT sensor was considered: the first model is a simple construction of magnetic system fig.2, and another model is an optimized design of magnetic system. The values of average magnetic flux density and heterogeneity of magnetic flux density were calculated at control zone.

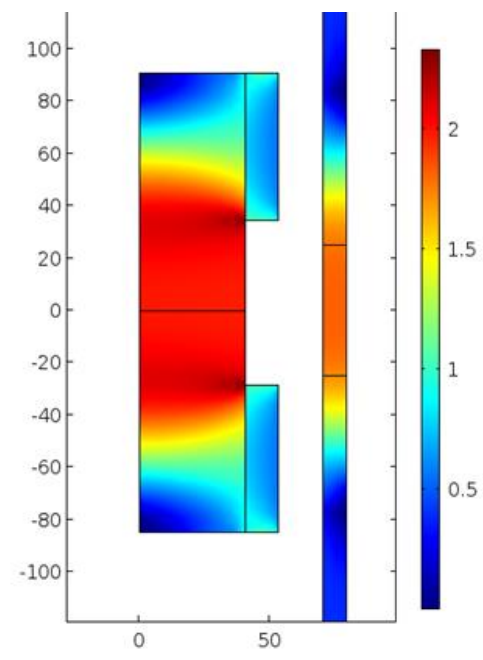

a)

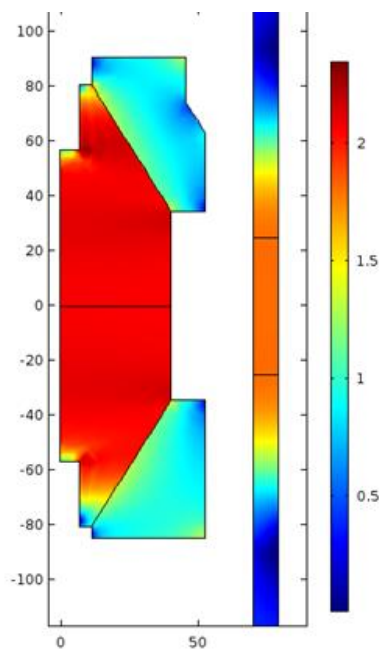

b)

Fig.3. Distribution of magnetic flux density at magnetic core and within pipe: (a)simple model; (b) optimized configuration

The longitudinal component of magnetic flux density within pipe at control zone (above the coil) was calculated. The value of average magnetic flux density $\mathrm{B}_{\mathrm{z}}$ was $1.77 \mathrm{Tl}$ and heterogeneity of magnetic flux density $\Delta \mathrm{B}_{\mathrm{z}}$ was $5.02 \%$ for the simple magnetic system model. Optimized system allowed achieving values of average magnetic flux density and heterogeneity of magnetic flux density, so the value $B_{z}$ was 1.80 and $\Delta B_{z}$ was $0.317 \%$. The homogeneity of the magnetic field is important because the presence of the different components magnetic flux density of is one of the design problems electromagnetic acoustic transducers [4].

\section{Displacement calculation}

To determine efficiency of this EMAT configuration, the mechanical displacement was calculated at COMSOL Solid Mechanics application. Further calculates the parameters of the sound wave to select the size of mesh for better convergence, so in this paper was considered EMAT configuration to produce SH sonic waves. Speed of sound transvers waves is $3000 \mathrm{~m} / \mathrm{s}$ for steel, wavelength according (3)

$$
\lambda=c / f
$$

c c - speed of sound, $\mathrm{f}-$ current frequency

Current pulse amplitude $50(\mathrm{~A})$ with frequency of $50(\mathrm{kHz})$ was used to drive the meander coil, maximum mesh size $\mathrm{X}$ was calculating according (4)

$$
x=\lambda / 25
$$

The r component of displacement filed $(u)$ are shown in Fig.4.

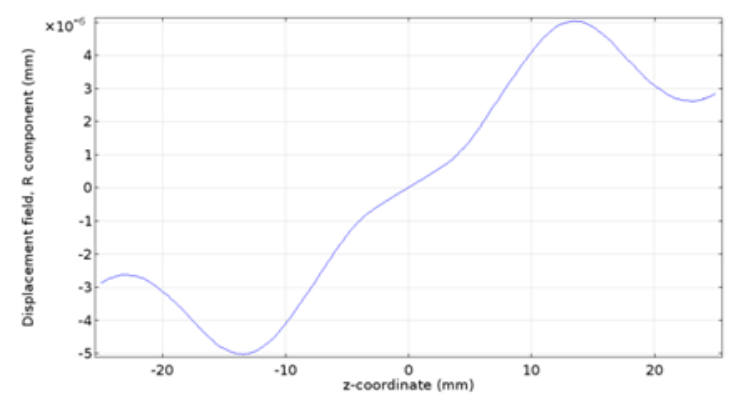

Fig.4 R-component of displacement field 
Because of the radial component of the magnetic flux density appears in the tube displacement in the longitudinal direction (z component of displacement field) see Fig.5.

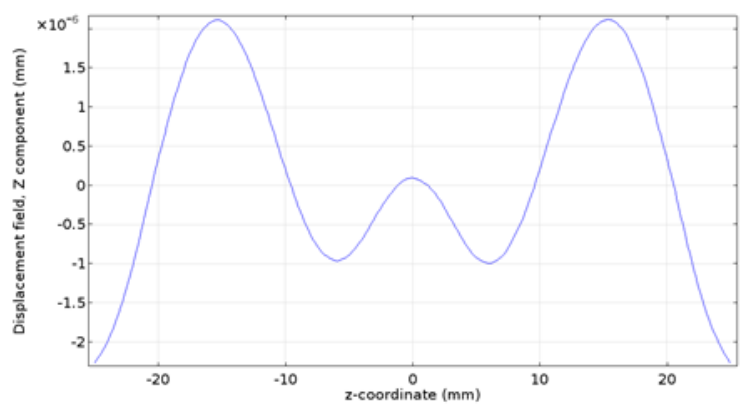

Fig.5 Z-component of displacement field

\section{Conclusion}

Mathematical model of electromagnetic acoustic transducer, using COMSOL multiphysics has been perfomed. EMAT technologies of non-destructive testing have a great potential for in-line inspection of small-size pipes. In future received signal modeling and manufacturing of the test piece.

\section{References}

[1] R. Ribichini, Modeling of electromagnetic acoustic transducers, thesis for the degree of doctor of philosophy, 2011

[2] S.Alekhin, Steel thickness measurement based on electromagnetic acoustic transducer with pulsed magnetic field, thesis thesis for the degree of doctor of philosophy , 2013.

[3] D,Reuter, T.Morgernstern, Ultrasound generation with high Power and coil only EMAT concept, Ultrasonics (54) (2014) 21141-2150.

[4] A. Samokrutov, S. Alekhin and et al, Modeling of EMAT magnetic systems for Ultrasonics waves generation in elastic Layer, in Static and Pulsed Magnetic fields, 11th European Conference on Non-Destructive Testing (ECNDT 2014), October 6-10, 2014, Prague, Czech Republic. 